\title{
ON THE COINCIDENCE OF THREE INVARIANT $\sigma$-ALGEBRAS ASSOCIATED WITH AN AFFINE TRANSFORMATION
}

\section{WILLIAM PARRY}

Introduction. This paper is concerned with an ergodic affine transformation $T$ of a compact metric connected abelian group $X . T$ is therefore a Haar measure preserving homeomorphism of $X$ onto itself and we can associate with $T$, three invariant $\sigma$-algebras or partitions $\pi, \alpha, \eta$ : these are the maximum $\sigma$-algebras such that the factors $T_{\pi}, T_{\alpha}, T_{\eta}$ have zero entropy, quasi-discrete spectrum, the distal property, respectively. It will be shown that the three $\sigma$-algebras are identical.

It is assumed that the reader is familiar with the theory of entropy of ergodic measure preserving transformations. For an account of this theory and its notations and for the related notions of measurable partition and factor transformation cf. [1].

Let $(X, \mathcal{E}, m)$ be a measure space point isomorphic to the unit interval with Lebesgue sets and Lebesgue measure. A compact metric abelian group with normalized complete Haar measure is of this type. Let $T$ be an invertible ergodic measure preserving transformation of $X$ on to itself. Associated with each sub- $\sigma$-algebra $D \subset \varepsilon$ there is an unique $(\bmod 0)$ measurable partition $\delta$ of $X$ and conversely. $(D=\varepsilon(\delta)$, $\delta=\epsilon(D)$.) $D$ is finite if and only if $\delta$ is finite. The relations $T D \mathcal{D}$, $T D=D$ correspond to the relations $T \delta \leqq \delta, T \delta=\delta(\bmod 0)$, and if $\nu, \Re, \epsilon$ are the trivial partition, trivial $\sigma$-algebra and partition into one point sets, respectively, then $\nu=\epsilon(\mathfrak{N}), \mathfrak{N}=\mathcal{E}(\nu), \epsilon=\epsilon(\mathcal{E}), \mathcal{E}=\mathcal{E}(\epsilon)$.

1. Corresponding to $T$ there is a maximum $\sigma$-algebra $\mathcal{E}(\pi) \subset \mathcal{E}$ such that the factor transformation $T_{\pi}$ has zero entropy. Moreover $T \&(\pi)$ $=\mathcal{E}(\pi) . T$ has zero entropy if and only if $\mathcal{E}(\pi)=\mathcal{E}$ and has completely positive entropy if and only if $\varepsilon(\pi)=\mathfrak{N}$. (Pinsker [2]).

2. If $\mathcal{E}(\gamma), \mathcal{E}(\delta)$ are doubly invariant $\sigma$-algebras $(T \mathcal{E}(\gamma)=\mathcal{E}(\gamma)$, $T \&(\delta)=\mathcal{E}(\delta)$,) and if $T_{\gamma}, T_{\delta}$ have zero entropy and completely positive entropy, respectively, then $\mathcal{E}(\gamma), \mathcal{E}(\delta)$ are independent i.e. if $A \in \mathcal{E}(\gamma)$, $B \in \mathcal{E}(\delta)$ then

$$
m(A \cap B)=m A \cdot m B \quad \text { [2]. }
$$

A homeomorphism $T$ of a compact metric space $X$ is said to be distal if $\lim _{m \rightarrow \infty} T^{n_{m}} x=\lim _{m \rightarrow \infty} T^{n_{m}} y$ implies $x=y$.

Received by the editors September 15, 1965. 
3. There is a minimum closed invariant equivalence relation on $X$ or, alternatively, there is a maximum closed Hausdorf partition $\eta$ of $X$ such that the factor transformation $T_{\eta}$ is distal (Ellis [3]).

4. Every homeomorphism which is a continuous image of a distal homeomorphism is distal [3].

An affine transformation $T$ of a compact abelian group $X$ is a transformation of the form

$$
T x=a+A(x),
$$

where $A$ is an automorphism of $X$ and $a \in X$.

For the definition of a measure preserving transformation with quasi-discrete spectrum cf. Abramov [4], and for the definition of a homeomorphism with quasi-discrete spectrum cf. [5].

An ergodic affine transformation $T$ of a compact metric connected abelian group $X$ possesses a maximum partition $\alpha$ such that $T_{\alpha}$ has quasi-discrete spectrum. The partition $\alpha$ is the same for both interpretations of "quasi-discrete spectrum," and $\varepsilon(\alpha)$ is the smallest $\sigma$-algebra for which the quasi-eigenfunctions are measurable.

5. $\alpha$ is the partition of $X$ into cosets of ann $\Gamma_{1}$, where ann $\Gamma_{1}$ is the annihilator of $\Gamma_{1}$ and $\Gamma_{1}$ is the subgroup of the character group $\Gamma$ given by

$$
\Gamma_{1}=\left\{\gamma \in \Gamma:(A-I)^{n} \gamma=0 \text { for some } n \geqq 1\right\} \quad[6] \text {. }
$$

We shall need the following result:

6. If $A$ is an automorphism of a compact abelian group (regarded as a Haar measure preserving transformation) such that all orbits (other than that of 0) are infinite, then $A$ has completely positive entropy. Under the same hypothesis, if $T x=a+A(x)$, then $T$ has completely positive entropy, for it is clear that $(A-I) X=X$ and if $(A-I) \beta=-a$ then

$$
T(\beta+x)=\beta+A(x),
$$

i.e. $T$ and $A$ are isomorphic [7].

In the next three statements we assume $T x=a+A(x)$ is an affine transformation of a compact metric connected abelian group $X$, regarded as a Haar measure preserving transformation.

7. $T$ is ergodic if and only if the smallest closed group containing a and $(A-I) X$ is $X$ and the only finite orbits in the character group $\Gamma$ under $A$ are fixed elements [8], [9].

8. If $T$ is ergodic and has quasi-discrete spectrum then $T$ has zero entropy [4]. 
9. If $T$ is ergodic and has quasi-discrete spectrum then $T$ is distal [5].

Throughout $T x=a+A(x)$ will be an ergodic affine transformation of a compact metric connected abelian group $X$, and $\Gamma$ will denote the character group of $X$. Automorphisms and their duals will be denoted by the same symbol. $Z(\lambda)$ will denote the ring of polynomials with integer coefficients.

Our results will depend on Propositions 1-9 and we refer to these propositions by ( ), whereas [ ] will denote a paper listed in the reference.

\section{Zero-entropy and quasi-discrete spectrum.}

THEOREM 1. The maximum partition $\pi$ such that $T_{x}$ has zero entropy and the maximum partition $\alpha$ such that $T_{\alpha}$ has quasi-discrete spectrum, concide.

Proof. In view of (5) and (8) it suffices to show that the partition of $X$ into cosets of ann $\Gamma_{1}$, where $\Gamma_{1}=\left\{\gamma \in \Gamma:(A-I)^{n} \gamma=0\right.$ for some $n \geqq 1\}$ is finer than $\pi$. Alternatively we have to show that the Fourier expansion of $\chi_{E}(x)$ does not depend on characters outside $\Gamma_{1}$, if $E \in \mathcal{E}(\pi)$.

Suppose $\gamma \notin \Gamma_{1}$ and suppose $p(A) \gamma \neq 0$ for all $0 \neq p \in Z(\lambda)$ then $[A, \gamma]$, the smallest $A$ invariant subgroup of $\Gamma$ containing $\gamma$, has no finite orbits other than the trivial orbit. $[A, \gamma]$ is the character group of $X^{\prime}=X / \operatorname{ann}[A, \gamma]$ and if $A^{\prime}$ is the induced automorphism on $X^{\prime}$ and $a^{\prime}$ is the coset of ann $[A, \gamma]$ to which $a$ belongs then $T^{\prime}\left(x^{\prime}\right)=a^{\prime}+A^{\prime}\left(x^{\prime}\right)$ has completely positive entropy (6). Consequently the coset partition of ann $[A, \gamma]$ is independent of $\pi$ and the Fourier expansion of $\chi_{E}(x)$ does not depend on $\gamma$, if $E \in \mathcal{E}(\pi)$. Therefore the partition into cosets of ann $\left(\Gamma^{\prime \prime}\right)$ where $\Gamma^{\prime \prime}=\{\gamma \in \Gamma: p(A) \gamma=0$ for some $0 \neq p \in Z(\lambda)\}$, is finer than $\pi$ and we may therefore suppose $\Gamma=\Gamma^{\prime \prime}$ i.e. we may suppose each $\gamma \in \Gamma$ is annihilated by some nontrivial $p(A)$ where $p \in Z(\lambda)$.

Let us embed $\Gamma$ into the smallest divisible group $\Gamma^{\prime}$ containing $\Gamma$. $\Gamma$ is countable and torsion free and therefore $\Gamma^{\prime}$ is countable and torsion free. Moreover for each $\gamma^{\prime} \in \Gamma^{\prime}$ there exists an integer $k \neq 0$ such that $k \gamma^{\prime} \in \Gamma$. The automorphism $A$ can be lifted to an automorphism $A^{\prime}$ of $\Gamma^{\prime}$ in such a way that if $\gamma^{\prime} \in \Gamma^{\prime}$ and $k$ is integer such that $k \gamma^{\prime} \in \Gamma$ then $k A^{\prime}\left(\gamma^{\prime}\right)=A\left(k \gamma^{\prime}\right)$.

Thus $T x=a+A(x)$ can be viewed as a factor of the affine transformation $T^{\prime} x^{\prime}=a^{\prime}+A^{\prime}(x)$, which acts on $X^{\prime}$, the character group of $\Gamma^{\prime}$, where $a^{\prime}$ is an element of the coset $a$ of $\operatorname{ann}(\Gamma)$.

Since $\Gamma$ has only fixed elements as finite $A$ orbits, the same is true of $\Gamma^{\prime}$ and $A^{\prime}$ orbits. To show that $T^{\prime}$ is ergodic we will show that the 
smallest closed group containing $a^{\prime}$ and $\left(A^{\prime}-I\right) X^{\prime}$ is $X^{\prime}(7)$. If this were not so there would exist a nontrivial character $\gamma^{\prime}$ such that $\left(A^{\prime}-I\right) \gamma^{\prime}=0$ and $\gamma^{\prime}\left(a^{\prime}\right)=0$ i.e. $\gamma^{\prime}\left(a^{\prime}\right)=0, \gamma^{\prime}\left(A^{\prime} x^{\prime}\right)=\gamma^{\prime}\left(x^{\prime}\right)$. But since there exists $k \neq 0$ such that $0 \neq \gamma=k \gamma^{\prime} \in \Gamma$ we would have $\gamma\left(a^{\prime}\right)=0$, $\gamma\left(A^{\prime} x^{\prime}\right)=\gamma\left(x^{\prime}\right), \gamma(A x)=\gamma(x)$. Moreover $\gamma$ is constant on the cosets of ann $\Gamma$ and therefore $\gamma(a)=0$. This contradicts the fact that the smallest closed subgroup of $X$ containing $a$ and $(A-I) X$ is $X,(7)$. $T$ is therefore a factor of the ergodic affine transformation $T^{\prime}$.

The character group $\Gamma^{\prime}$ of $X^{\prime}$ is divisible and for every $\gamma^{\prime} \in \Gamma^{\prime}$ there exists $0 \neq p \in Z(\lambda)$ such that $p\left(A^{\prime}\right) \gamma=0$. Write $p(\lambda)=(\lambda-1)^{n} p^{\prime}(\lambda)$ where $p^{\prime} \in Z(\lambda)$ and $p^{\prime}(\lambda), \lambda-1$ are coprime. Let $u, v \in Z(\lambda)$ be such that

$$
u(\lambda)(\lambda-1)^{n}+v(\lambda) p^{\prime}(\lambda)=k
$$

for some integer $k \neq 0$, then

$$
u\left(A^{\prime}\right)\left(A^{\prime}-I\right)^{n}\left(\gamma^{\prime} / k\right)+v\left(A^{\prime}\right) p^{\prime}(A)\left(\gamma^{\prime} / k\right)=\gamma^{\prime} .
$$

Therefore $\gamma^{\prime}=\gamma_{1}^{\prime}+\gamma_{2}^{\prime} \quad$ where $\gamma_{1}^{\prime}=v\left(A^{\prime}\right) p^{\prime}(A)\left(\gamma^{\prime} / k\right), \quad \gamma_{2}^{\prime}=$ $u\left(A^{\prime}\right)\left(A^{\prime}-I\right)^{n}\left(\gamma^{\prime} / k\right)$ and $p^{\prime}\left(A^{\prime}\right) \gamma_{2}^{\prime}=0,\left(A^{\prime}-I\right)^{n} \gamma_{1}^{\prime}=0$.

Let $\Gamma_{2}^{\prime}=\left\{\gamma^{\prime} \in \Gamma^{\prime}: p^{\prime}\left(A^{\prime}\right) \gamma^{\prime}=0\right.$ for some $p^{\prime}(\lambda)$ prime to $\left.\lambda-1\right\}$ and let $\Gamma_{1}^{\prime}=\left\{\gamma^{\prime} \in \Gamma^{\prime}:\left(A^{\prime}-I\right)^{n} \gamma^{\prime}=0\right.$ for some $\left.n \geqq 1\right\}$. $\Gamma_{1}^{\prime}, \Gamma_{2}^{\prime}$ are $A^{\prime}$ invariant subgroups of $\Gamma^{\prime}$ and $\Gamma^{\prime}=\Gamma_{1}^{\prime} \times \Gamma_{2}^{\prime}$.

Consequently $X^{\prime}=X_{1}^{\prime} \times X_{2}^{\prime}$ where $X_{1}^{\prime}=X^{\prime} /$ ann $\Gamma_{1}^{\prime} X_{2}^{\prime}=X^{\prime} /$ ann $\Gamma_{2}^{\prime}$ and the character groups of $X_{1}^{\prime}, X_{2}^{\prime}$ are $\Gamma_{1}^{\prime}, \Gamma_{2}^{\prime}$, respectively. Moreover $T^{\prime}=T_{1}^{\prime} \times T_{2}^{\prime}$ where $T_{1}^{\prime}\left(x_{1}^{\prime}\right)=a_{1}^{\prime}+A_{1}^{\prime}\left(x_{1}^{\prime}\right), T_{2}^{\prime}\left(x_{2}^{\prime}\right)=a_{2}^{\prime}+A_{2}^{\prime}\left(x_{2}^{\prime}\right)$ and $A_{1}^{\prime}, A_{2}^{\prime}$ are the automorphisms induced on $X_{1}^{\prime}, X_{2}^{\prime}$ by $A^{\prime}$, respectively, and $a_{1}^{\prime}, a_{2}^{\prime}$ are the cosets of $\operatorname{ann}\left(\Gamma_{1}^{\prime}\right)$, ann $\left(\Gamma_{2}^{\prime}\right)$ of $X^{\prime}$, respectively, to which $a^{\prime}$ belongs.

From (5), (8) it follows that $T_{1}^{\prime}$ has quasi-discrete spectrum and zero entropy. From (6) it follows that $T_{2}^{\prime}$ has completely positive entropy. These two statements together with (2) imply that $\pi^{\prime}$ (the maximum partition of $X^{\prime}$ with zero entropy for $T^{\prime}$ ) is the partition of $X^{\prime}$ into cosets of ann $\Gamma_{1}^{\prime}$.

But $\pi \leqq \pi^{\prime}$ and if $(E, X-E) \leqq \pi$ the Fourier expansion of $\chi_{E}\left(x^{\prime}\right)$ depends only on the characters of $\Gamma \cap \Gamma_{1}^{\prime}$. Consequently the Fourier expansion of $\chi_{E}(x)$ depends only on the characters of

$$
\Gamma_{1}=\left\{\gamma \in \Gamma:(A-I)^{n} \gamma=0 \text { for some } n \geqq 1\right\} \text {, }
$$

and the proof is complete.

2. The distal property and quasi-discrete spectrum. 
THEOREM 2. The maximum partition $\eta$, such that $T_{\eta}$ is distal and the maximum partition $\alpha$ such that $T_{\alpha}$ has quasi-discrete spectrum, coincide.

Proof. In view of (9) it suffices to show that $T_{\eta}$ has quasi-discrete spectrum. We first note that $\eta$ is translation invariant by showing that $T_{\eta+x}$ is also distal for each $x \in X$. Let $d$ be a translation invariant metric on $X$. Since the distal relation is closed and since $X$ is compact metric, it follows that the factor space $X_{\eta}$ is also compact metric, $\left[10\right.$, p. 148]. Let $d^{\prime}$ be a metric for $X_{\eta}$, then

$$
\lim _{m \rightarrow \infty} d^{\prime}\left(T_{\eta}^{n_{m}} E, T_{\eta}^{n_{m}} F\right)=0
$$

if and only if there exist $x_{m} \in E, y_{m} \in F$ such that

$$
\lim _{m \rightarrow \infty} d\left(T^{n_{m}} x_{m}, T^{n_{m}} y_{m}\right)=0 .
$$

Consequently, if $x_{m} \in E \in \eta, y_{m} \in F \in \eta$ then

$$
\begin{aligned}
\lim _{m \rightarrow \infty} d\left(T^{n_{m}} x_{m}, T^{n_{m}} y_{m}\right) & =\lim _{m \rightarrow \infty} d\left(A^{n_{m}} x+T^{n_{m}} x_{m}, A^{n_{m}} x+T^{n_{m}} y_{m}\right) \\
& =\lim _{m \rightarrow \infty} d\left(T^{n_{m}}\left(x_{m}+x\right), T^{n_{m}}\left(y_{m}+x\right)\right) \\
& =0
\end{aligned}
$$

implies $E=F$. Therefore $\eta+x \leqq \eta$ for all $x \in X$, and $\eta$ is a partition of $X$ in to cosets of some closed subgroup $X^{\prime}$ of $X$. We may therefore suppose that $T$ is a distal ergodic affine transformation and prove that $T$ has quasi-discrete spectrum.

In view of (5) we have to show that for each $\gamma \in \Gamma$, there exists $n \geqq 1$ such that $(A-I)^{n} \gamma=0$. If this were not so, then either there would exist $\gamma \in \Gamma$ and $p \in Z(\lambda)$ prime to $\lambda-1$ such that $p(A) \gamma=0$ or there would exist $\gamma \in \Gamma$ such that for all $0 \neq p \in Z(\lambda), p(A) \gamma \neq 0$. In either case (using (7) and the ergodicity of $T$ ) $A$ would have no finite orbits in $[A, \gamma]$ other than that of $0 .[A, \gamma]$ is the character group of $X^{\prime}=X / \operatorname{ann}[A, \gamma]$ and if $T^{\prime} x^{\prime}=a^{\prime}+A^{\prime}\left(x^{\prime}\right)$ where $A^{\prime}$ is the automorphism induced on $X^{\prime}$ by $A$ and $a^{\prime}$ is the coset of ann $[A, \gamma]$ to which $a$ belongs, then $T^{\prime}$ is a continuous image of $T$. Consequently $T^{\prime}$ is distal, and since $T^{\prime}$ is conjugate to $A^{\prime}$ cf. (6), $A^{\prime}$ is distal. Since $A^{\prime}$ is ergodic, for almost all $x^{\prime} \in X^{\prime}$ there exist a sequence $\left\{n_{m}\right\}$ such that

$$
\lim _{m \rightarrow \infty} A^{\prime n} n_{m}\left(x^{\prime}\right)=0,
$$

and obviously $\lim _{m \rightarrow \infty} A^{\prime n_{m}}(0)=0$. 
Therefore $A^{\prime}$ is not distal. This contradiction establishes the theorem.

\section{REFERENCES}

1. V. A. Rohlin, New progress in the theory of transformations with invariant measure, Uspehi Mat. Nauk. 15 (1960), 3-26= Russian Math. Surveys 15 (1960), 1-22.

2. M. S. Pinsker, Dynamical systems with zero or completely positive entropy, Dokl. Akad. Nauk. SSSR 133 (1960), 1025-1026=Soviet Math. Dokl. 1 (1960), 937-938.

3. R. Ellis and W. H. Gottschalk, Homomorphisms of transformation groups, Trans. Amer. Math. Soc. 94 (1960), 258-271.

4. L. M. Abramov, Metric automorphisms with quasi-discrete spectrum, Izv. Akad. Nauk. SSSR Ser. Mat. 26 (1962), 513-530; Amer. Math. Soc. Transl. (2) 39 (1964), $37-56$.

5. F. Hahn and W. Parry, Minimal dynamical systems with quasi-discrete spectrum, J. London Math. Soc. 40 (1965), 309-323.

6. H. Hoare and W. Parry, Affine transformations with quasi-discrete spectrum, J. London Math. Soc. 41 (1966), 88-96.

7. V. A. Rohlin, Metric properties of endomorphisms of compact commutative groups, Izv. Akad. Nauk SSSR Ser. Mat. 28 (1964), 867-874.

8. F. Hahn, On affine transformations of compact abelian groups, Amer. J. Math. 85 (1963), 428-446.

9. H. Hoare and W. Parry, Semi-groups of affine transformations, Oxford Quart. Math. 17 (1966), 106-111.

10. J. L. Kelley, General topology, Van Nostrand, New York, 1955.

University of Sussex, England 